\title{
An experimental study on fine structures of supersonic laminar/ turbulent flow over a backward-facing step based on NPLS
}

\author{
CHEN Zhi ${ }^{*}$ YI ShiHe, HE Lin, TIAN LiFeng \& ZHU YangZhu \\ College of Aerospace and Material Engineering, National University of Defense Technology, Changsha 410073, China
}

Received July 5, 2011; accepted September 2, 2011

\begin{abstract}
Fine structures of supersonic flow over a $5 \mathrm{~mm}$ high backward facing step (BFS), including expansion wave fan, reattachment shock, supersonic boundary layer were measured in a $M a=3.0$ low-noise indraft wind tunnel. By varying the superficial roughness of the wall upstream from the step, supersonic laminar flow and supersonic turbulent flow could be formed over a BFS. Measurements on the spatiotemporal features of the holistic flow field and the fine structures in four typical regions were carried out using NPLS (nano-based planar laser scattering). Flow structures, including expansion wave fan, reattachment shock, supersonic boundary layer and its separation, reattachment and redevelopment are revealed by measuring the holistic structure of the transient flow field. Comparing the two time-averaged flow fields with each other, it is apparent that supersonic turbulent flow over a BFS (STF-BFS) has a larger expansion angle and a shorter recirculation region, and its redeveloped boundary layer increases at a smaller obliquity while the angle of reattachment shock is the same for the supersonic laminar flow over a BFS (SLF-BFS). With regard to time-evolution features, the $K-H$ vortices in the SLF-BFS suffers from shearing, expansion, reattachment and three-dimensional effects while in the STF-BFS large-scale structures are affected by the incline and distortion at the reattachment point due to expansion, viscosity and reverse-pressure. Studies on local regions indicate that in the SLF-BFS, the emergence of compression waves which distinctly converge into a reattachment shock is due to the local convective Mach number and the inducement of $K-H$ vortices in the free shear layer. Nevertheless, in the STF-BFS, compression waves and $K-H$ vortices are barely evident, and the formation of a reattachment shock is related to the wall compressive effect.
\end{abstract}

supersonic flow over a backward facing step, nano-based planar laser scattering, fine structure, laminar, turbulent

Citation: Chen Z, Yi S H, He L, et al. An experimental study on fine structures of supersonic laminar/turbulent flow over a backward-facing step based on NPLS. Chin Sci Bull, 2012, 57: 584-590, doi: 10.1007/s11434-011-4888-y

As a typical flow with simple geometrical boundaries, flow over a backward facing step (BFS) contains many complicated structures, including separation and reattachment. Separated/reattaching flows often occur in natural environments such as air movement in valleys or over fences as well as in many man-made facilities such as airfoils with an attack angle, reentry aircrafts, diffusers, and turbo engines. However, generally speaking, separation results in negative effects on these systems, e.g. reducing manipulation of aircraft, increasing heat loss from walls, creating noise and dynamic structural loads. However, in the research field of supersonic flows, the BFS is always adopted as a typical

*Corresponding author (email: gfkdchenzhi@163.com) configuration for ignition in a scramjet, where the recirculation region has an important role in stabilizing the firing of the engine. Steps on the surface of supersonic/hypersonic aircrafts make flow fields complex, and consequently appropriate research is propitious for optimizing the dynamic design of aircraft.

In the past several decades, a lot of experimental and numerical work has been done on BFS flows, focusing on the flow mechanism, controlling effect and unsteadiness. To study flow structures using flow visualization, schlieren, shadow, planar laser scattering techniques, white-light bubble image velocimetry and computational fluid dynamics methods are usually adopted. Shen and Ma [1] used white-light bubble image velocimetry to measure the struc- 
tures of starting vortices in a water tunnel. They found four stages in this process: (1) The first acceleration stage; (2) the second acceleration stage; (3) the secondary induced vortex and second separated vortex stage; (4) the breakdown stage. The starting vortex was much stronger than the following shedding vortices. Using laser velocimetry, Cohen and Bennett [2] measured the velocity field behind a backward-facing step disturbed by a certain frequency. The results showed that the recirculation region grew and shrank periodically with the change in disturbing frequency, which revealed its deviation from a quasi-steady representation. Large-scale structures in a two-dimensional BFS flow were studied by Noriyuki et al. [3] using multi-point laser Doppler velocimetry. A space and time correlation and conditional average were adopted to analyze the moving path of the shedding vortex. The large-scale fluctuation was proposed as a self-excitation motion. Tinney and Ukeiley [4] performed an investigation into the flow over a threedimensional double BFS using oil-flow visualization and particle image velocimetry. It was found that there were some similar features in both three-dimensional and twodimensional flows. The development of horseshoe vortices was introduced by the geometry of the three-dimensional model. The quasi-periodic shedding of the horseshoe vortices was thought to cause the large turbulent fluctuation. A numerical study on passive control of BFS flow was carried out by Neumann and Wengle [5]. Flow over a BFS with a surface-mounted control fence was simulated by direct numerical simulation and large-eddy simulation resulting in an understanding of the controlling effect at $R e_{h}=3000$. Results were in good agreement with each other and showed that there was a certain minimum distance between the step edge and the position of the control fence for achieving a maximum reduction of the reattachment length.

Traditional flow visualization techniques such as smokewire technique and hydrogen bubble techniques are mainly used in experimental research on low-speed gas and water. Schlieren, shadow and interference methods, which can realize non-intrusive measurements on flow structures, encountered the problems of integral effects and low spatial resolution, and are not suitable for measuring the complicated structures of high speed turbulence that requires high spatiotemporal resolution [6]. In addition, planar laser induced fluorescence and filtered Rayleigh scattering are inadequate for low scattering signals and low signal-to-noise ratios (SNRs). Nano-based planar laser scattering (NPLS) is a new flow visualization technique for measuring fine structures of supersonic/hypersonic flow. Its spatial resolution can reach the micrometer scale, with a time resolution of $6 \mathrm{~ns}$, and the temporal correlation resolution can reach $0.2 \mu \mathrm{s}[7,8]$.

In this paper, NPLS is adopted to visualize the supersonic laminar flow over a BFS (SLF-BFS) and supersonic turbulent flow over a BFS (STF-BFS). The holistic structures of these flow fields and the fine structures of four typical local regions are presented. The spatiotemporal evolution features of the transient flow fields and the time-averaged flow structures are studied and analyzed. Discrepancy of these two flows are discussed in terms of the boundary layer, free shear layer and compression wave, length of recirculation region, location of reattachment point, and recovery of the redeveloped boundary layer.

\section{Experimental setup}

\subsection{NPLS technique}

NPLS, which solves the problem of particles' following ability in a supersonic/hypersonic flow field, is a visualization technique for measuring fine flow structures in high speed and complicated flow fields using nanoparticles for tracing. As shown in Figure 1, the NPLS system used in this study is composed of light source, imaging, restoring and controlling systems. A dual-cavity Nd: YAG pulsed laser is used as the light source, which emits two laser beams of 6 ns pulse width according to the schedule set by a synchronizer. A light sheet, less than $1 \mathrm{~mm}$ thick, illuminates the flow field of interest. Because of the excellent following ability of nanoparticles, they can cope with complicated structures in supersonic flow fields and scatter laser light effectively to generate high SNR images [8-11]. An interline transfer double-exposure CCD, whose resolution is $2 \mathrm{k} \times 2 \mathrm{k}$ with a shortest double-exposure interval of $0.2 \mu \mathrm{s}$, takes charge of imaging. The synchronizer, which has an accuracy of $250 \mathrm{ps}$, can adjust the time when the laser emits and the CCD takes exposures according to signals from the computer; thus ensuring that the two laser beams are exposed in the frames of dual-exposure. The computer is used to set the parameters of the synchronizer, storing and processing images.

\subsection{Supersonic wind tunnel and the testing model}

Measurements on flow structures of supersonic BFS flow were carried out in a $M a=3.0$ low-noise wind tunnel which ran in an indraft mode. The incoming flow was dried and dust free, and the total pressure and stagnation temperature

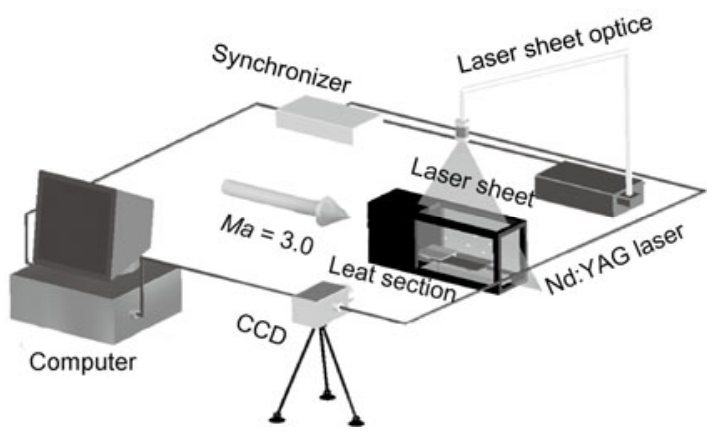

Figure 1 NPLS testing system. 
were $P_{0}=1$ atm and $T_{0} \approx 300 \mathrm{~K}$. The nozzle was designed based on a B-spline curve which eliminated waves in the expansion section of the nozzle. Results from numerical simulation and experimental testing showed that a nozzle designed by this method could generate a supersonic flow field suitable for measuring fine structures in turbulence $[12,13]$. As shown in Figure 2, the cross section of the testing chamber was a $100 \mathrm{~mm} \times 120 \mathrm{~mm}$ rectangle, and the dimensions of its two side faces encased with high quality optical glass for imaging were $250 \mathrm{~mm} \times 120 \mathrm{~mm}$.

The testing model for a 2D BFS is shown in Figure 3, for which the step height $h$ was $5 \mathrm{~mm}$, the spanwise length $d$ was $120 \mathrm{~mm}$, and the distance between the wall downstream the step and the ceiling of the testing chamber $H$ was $80 \mathrm{~mm}$. These parameters, as shown in Table 1 , ensured a $d / h \geqslant 10$ and an expansion ratio of $E r=1.067$, which enabled the flow to be considered two-dimensional. By changing the surface roughness of the wall upstream step, supersonic laminar and turbulent flows can be generated.

\section{Visualization and analysis of fine flow struc- tures}

\subsection{Flow structures of the SLF-BFS}

According to the text above, the high SNR NPLS image is generated by gathering the light scattered by tracing nanoparticles in the testing flow field. The excellent following ability of the particles ensures that the distribution of the scattering light can reveal complicated flow structures. As shown in Figure 4, it is the NPLS image of the SLF-BFS transient flow field, which distinctly reveals the structures of the whole flow field and its evolution. Light from the

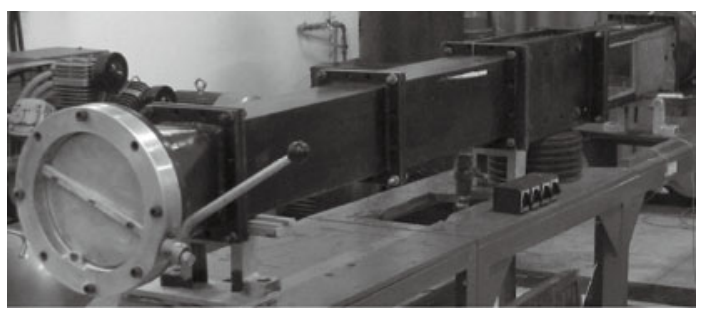

Figure $2 M a=3.0$ low noise supersonic wind tunnel.

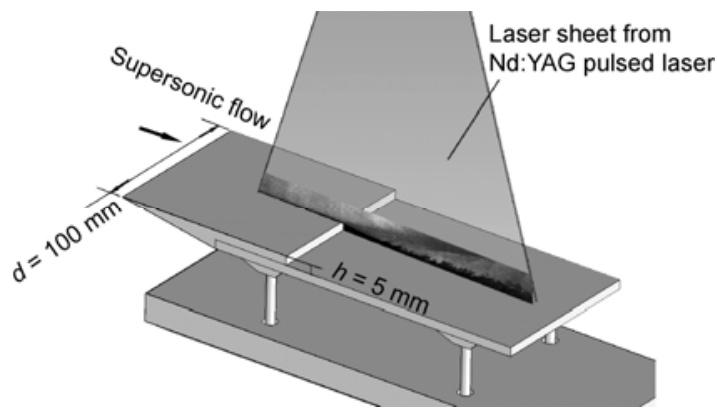

Figure 3 Testing model of the two-dimensional BFS. main flow region is scattered evenly because the nanoparticles are distributed uniformly. However, scattered light from the boundary layer and recirculation region is weak due to fewer nanoparticles in these regions. As shown in Figure 4, the supersonic laminar boundary layer forms on the wall upstream from the step and its thickness increases slowly along the streamwise direction. After passing over the step, it accelerates while its density decreases because of the sudden geometric expansion of the wall and the impact from the expansion waves. The region of expansion waves in Figure 4 is relatively darker. A free shear layer is formed between the separated flow and the recirculation region at the corner of the step. Several compression waves, which, based on large numbers of experimental results, are thought to be induced by the $K-H$ vortices in the free shear layer, converge into a reattachment shock. Reattachment occurs somewhere downstream from the step generating a recirculation region between the reattachment point and the step. There is a distinct development of a new boundary layer after reattachment with its thickness increasing and structures evolving rapidly.

By repeating measurements and performing an averaging process, a time-averaged flow field can be obtained, as shown in Figure 5. Although some transient characters are blotted out in the averaging process, some flow unsteadiness is also eliminated, which is convenient for acquiring and analyzing the time-averaged structural features. From Figure 5 it can be seen that the supersonic laminar boundary layer is thin and hardly increases within the $\Delta X=5 h$ distance upstream from the step, compared with the rapid increase of the redeveloped supersonic turbulent boundary layer. Nevertheless, the structure of the free shear layer generated by separation is heavily obliterated while structures of the expansion wave fan and reattachment shock are more evident. Structures of the boundary layer, expansion region, and reattachment shock in this figure can be identified using the image analyzing technique based on the grayscale variation and Canny edge detection technique [13]. Measurements show that the angle of expansion wave fan is $25.5^{\circ}$, while that of reattachment shock is $13.4^{\circ}$ and the redeveloping supersonic turbulent boundary layer increases at an angle of $4.6^{\circ}$, indicating that the separated supersonic laminar boundary layer is unstable and can easily change into turbulence.

\subsection{Flow structures of STF-BFS}

Figure 6 shows the transient flow field of the STF-BFS. As the dependent region of the flow field downstream, the supersonic turbulent boundary layer upstream from the step greatly affects the structures of separation, reattachment and redevelopment, which results in a distinct discrepancy to the SLF-BFS flow field. First, a supersonic turbulent boundary layer is thicker than a laminar one as shown in Figure 4 and creates structural irregularity and intensive fluctuations and 
Table 1 Parameters of the 2D BFS testing model

\begin{tabular}{ccccccccc}
\hline$h$ & $d$ & $d / h$ & $H$ & $E r=H /(H-h)$ & $M a_{\infty}$ & $T_{0 \infty}$ & $P_{0 \infty}$ \\
\hline $5 \mathrm{~mm}$ & $120 \mathrm{~mm}$ & 24 & $80 \mathrm{~mm}$ & 1.067 & 3.0 & $300 \mathrm{~K}$ & $1 \mathrm{~atm}$ & $3.85322 \times 10^{4}$ \\
\hline
\end{tabular}

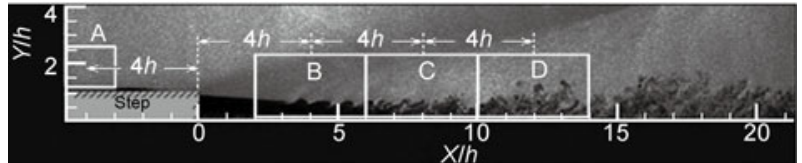

Figure 4 NPLS image of the SLF-BFS transient flow field.

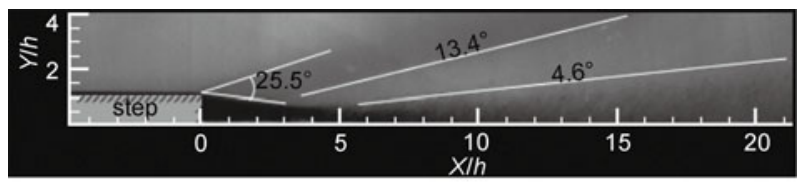

Figure 5 Time-averaged flow field of the SLF-BFS.

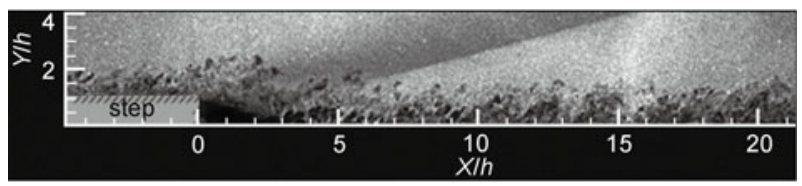

Figure 6 NPLS image of the STF-BFS transient flow field.

transport in the whole flow field. It greatly contrasts with the separated boundary layer in Figure 4 which is an unstable and transition process. Second, by comparing Figures 4 and 6 , it can be seen that the recirculation region is shorter in the STF-BFS flow field with a reattachment point much closer to the step. Third, with regard to the supersonic turbulent flow field in Figure 6, no $K-H$ vortices appear in the free shear layer, but the angle of the shear layer and the lower wall of the model is larger. These three points indicate that supersonic turbulence is hard to separate and more stable when it suffers from the effect of geometric variety.

The results of measurements on the time-averaged flow field of the STF-BFS, as shown in Figure 7, reveal that the angle of the expansion wave fan increases to $34.6^{\circ}$ due to the decrease in the recirculation region, while the reattachment shock is still $13.4^{\circ}$ inclined to the wall. This is the same as in Figure 5, which means the reattachment shock is related to the compression effect of the wall downstream from the step. However, the redeveloped boundary layer in this condition increases by an angle of only $1.86^{\circ}$.

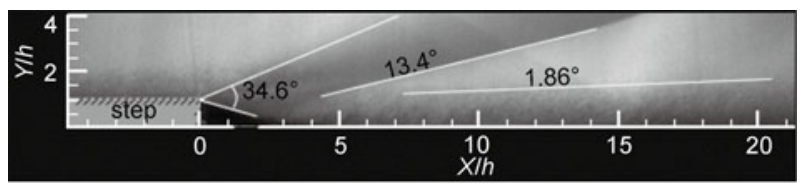

Figure 7 Time-averaged flow field of the STF-BFS.

\subsection{Time-evolution of flow structures}

The time-evolution of the SLF-BFS flow field is revealed in Figure 8 where the interval between the two figures is $10 \mu \mathrm{s}$. Structures at three typical locations are selected and compared. Structure A at $t_{1}$ is the state of separated free shear when it changes from laminar flow into $K-H$ vortices. After $10 \mu$ s development, as the arrow ' $a$ ' reveals at $t_{2}$, this structure mainly suffers from shearing distortion with its movement downstream, shown by its transformation. Structure B is the more fully developed $K-H$ vortices after a distance of $3 h$ compared with Structure A. Because of the expansion effect caused by the movement of the shear layer inclined to the wall and the impact of reattachment, the corresponding structure ' $\mathrm{b}$ ' at $t_{2}$, which is similar to the streamwise structure of the $\Lambda$ vortex in the supersonic flat-plate boundary layer [14], expands to a certain extent because of a threedimensional effect [4]. In addition, the transformation of the $K-H$ vortices induces the variation in compression waves. The redeveloped turbulent boundary layer after reattachment as shown at ' $C$ ' and ' $c$ ', mainly represents the vortex transformation caused by the high frequent fluctuations and transport.

Compared with the SLF-BFS, the time-evolution of the STF-BFS flow field is characteristic for an inclined structure around the reattachment point as revealed by ' $A$ ' and ' $B$ ' in Figure 9. There are two main reasons: the near wall region of the structure after reattachment suffers from the viscosity and converse pressure effects along the streamwise direction; and the upside part of the structure accelerates due to the effect of expansion waves upstream.

\subsection{Fine flow structures in four typical regions}

Four typical regions of $4 h$ width are selected to be measured and studied further, as shown by A, B, C and D in Figure 4. Region A is located at a $4 h$ distance upstream from the step,

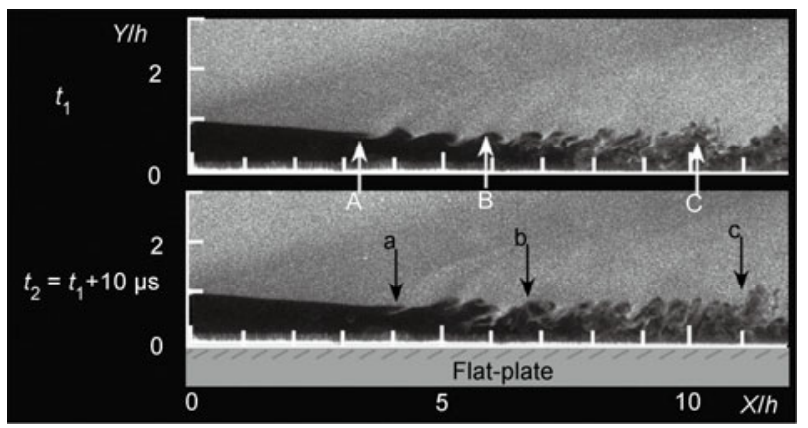

Figure 8 Time-evolution of the SLF-BFS flow field. 


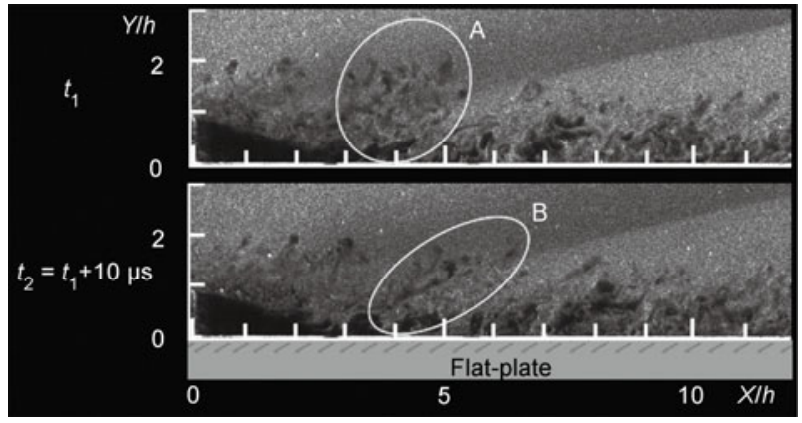

Figure 9 Time-evolution of the STF-BFS flow field.

where the supersonic flat-plate boundary layer is the main structure and impacts the other regions downstream, thus it cannot be neglected. It is convenient for comparison that the $4 h$ wide testing range is determined according to the requirements of the numerical simulation and matches with most former studies. The center of Region B is located $4 h$ downstream from the step covering most of the recirculation region. In fact, this region contains the most complicated structures which are the main points in the studies on BFS flow. Scattered light from the step corner is intense and greatly affects the experiments. Thus, to avoid it, Region B is moved downstream and does not cover the whole recirculation region. Although some structures that deserved to be studied were lost because of this experimental configuration, the basic features of the recirculation region are still contained in Region B. As for Region C, the reattachment occurs $8 h$ downstream from the step. Studies on incompressible BFS flow generally consider that the reattachment point is at the location of $X=7 h$. The last Region D is moved $12 h$ downstream from the step, where the new boundary layer redevelops after reattachment. As impacts from the other regions upstream are revealed synthetically in Region D, it is usually selected as an important region for testing the controlling effect upstream. The fine structures of these testing regions are shown in Figures 10-13.

(i) Boundary layer on the wall upstream from the step. Figure 10 reveals the fine structures of the supersonic laminar (a) and turbulent (b) boundary layers which form on the flat-plate surface upstream from the step. It can be established that in Region A the thickness of the laminar boundary layer is about $0.1 \mathrm{~h}$ which develops slowly and stably without any distinct structures. However, the turbulent structure is $1 h$ thick with small-scale vortex structures seen clearly in the figure due to its intense fluctuation, which results in more nanoparticles in the turbulent boundary layer.

(ii) Recirculation region. Figure 11 depicts structures such as the recirculation region, free shear layer and compression waves. As for the transient flow field on the left in Figure 11, the supersonic boundary layer, which comes from Region A upstream, remains laminar within the range of $X<3 h$ after its separation from the step. With its movement towards the wall downstream, the shearing extent
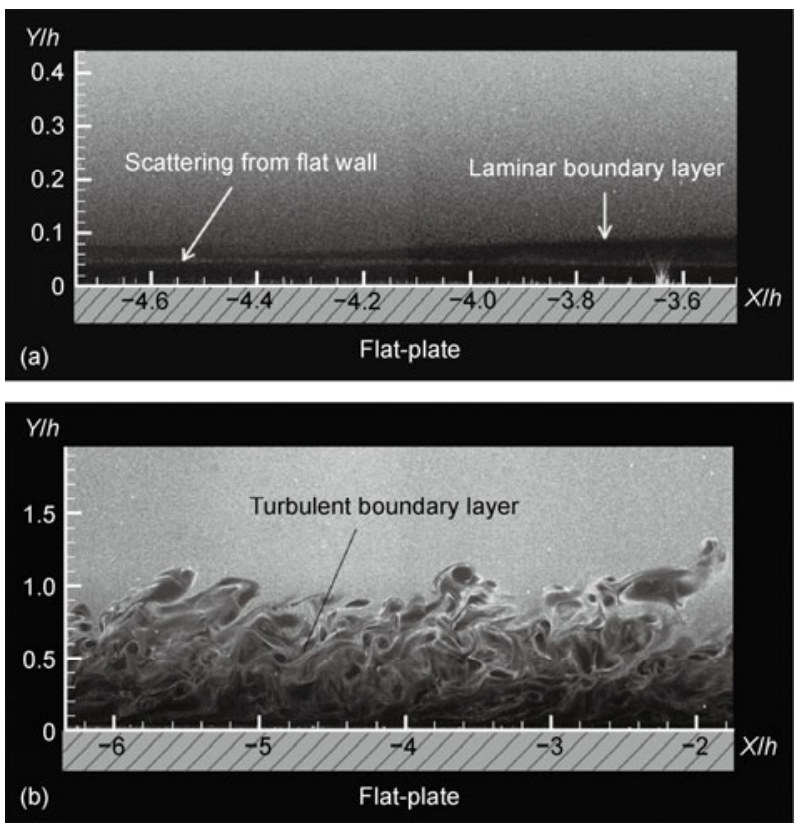

Figure 10 Boundary layer upstream from the step in SLF-BFS (a) and STF-BFS (b), corresponding to Region A in Figure 4.
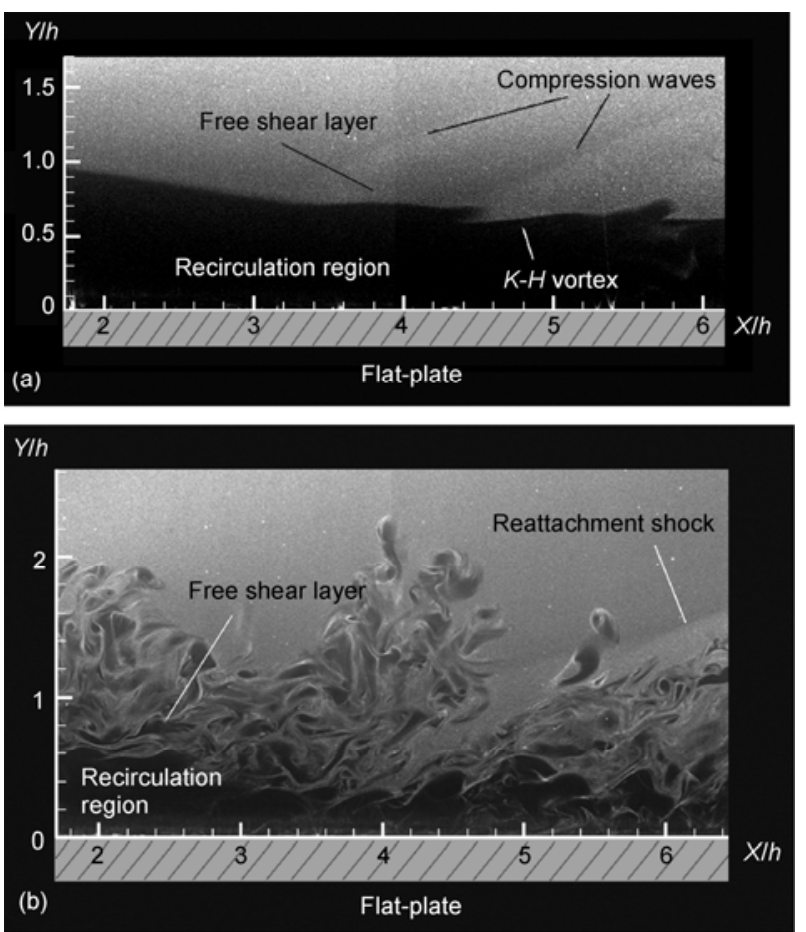

Figure 11 Flow structures around the recirculation region in SLF-BFS (a) and STF-BFS (b), corresponding to Region B in Figure 4.

between this separated flow and the recirculation region increases, and $K-H$ vortices gradually appear after $X>3 h$. Reattachment occurs downstream after supersonic flow separates. The range from the step point to the reattachment point is the recirculation region for which the length is determined by quantitative measurements. In this case, it can be judged that the supersonic laminar flow reattaches 
somewhere out of Region B, and its location $X_{\mathrm{r}}$ is more than $6 h$. In addition, the intensity of compression waves generated by the SLF-BFS flow is weak, which can be vaguely discerned in the figure. These waves will converge into a reattachment shock with its radiation downstream. Note that the generation of compression waves relates to the $K-H$ vortices in the shearing layer.

The flow field of the STF-BFS is shown in Figure 11(b). Even though it is in the same Region B as the SLF-BFS, its structures differ due to a short recirculation region. Weak signals at $X=3 h-4 h$ near the wall reveal the approximate reattachment location. Recirculation and the shearing layer are not distinct in this testing region, but according to the location of the reattachment point the length of the recirculation region is determined to be $3 h-4 h$. The separated turbulent boundary layer collides on the wall after reattachment, generating a ridge-like structure. A distinct reattachment shock occurs at $X=6 h$, but without the convergence of several compression waves into the reattachment shock.

(iii) Reattachment region. It is considered that reattachment occurs at the location of $X=7 h$ downstream from the step according to former studies on incompressible flows. The so-called reattachment region is actually the range where reattachment point locates and wanders along the stream due to flow instability. Although the location of reattachment needs to be determined by quantitative analysis, it can still be shown qualitatively that the reattachment is located at $X=7 h-7.5 h$ measured by NPLS as indicated in Figure 12(a). This is because the shearing layer terminates here according to the analysis based on the grayscale distribution. The redeveloped boundary layer rapidly experiences transition with its structures varying acutely at $X=7.5 h-10 h$. In addition, the phenomenon of compression waves converging into a reattachment shock becomes more evident because of the compression effect of the wall when the separated supersonic flow impinges on it.

According to the test above, reattachment occurs in Region B for the STF-BFS flow in Figure 12(b), and thus the reattachment shock and redeveloped boundary layer are the main structures here in Region C. The reattachment shock is more intense and the new turbulent boundary layer develops without an obvious increase, but with vortices created by cracking.

(iv) Recovery region after reattachment. The main flow structure downstream after reattachment is the redeveloped supersonic boundary layer. Flow structures of the recovery region in the SLF-BFS are shown in Figure 13(a). The redeveloped boundary layer changes into supersonic turbulence but is not fully developed. Comparing it with Figure 11(a) and Figure 12(a), the supersonic laminar boundary layer experiences an obvious process including separation, reattachment and recovery with its structure evolving and thickness increasing because of the step. At the location $X=14 h$ this supersonic turbulent boundary layer is $1.0 \mathrm{~h}$ thick and 10 times that in Region A. Thus the effect of separation
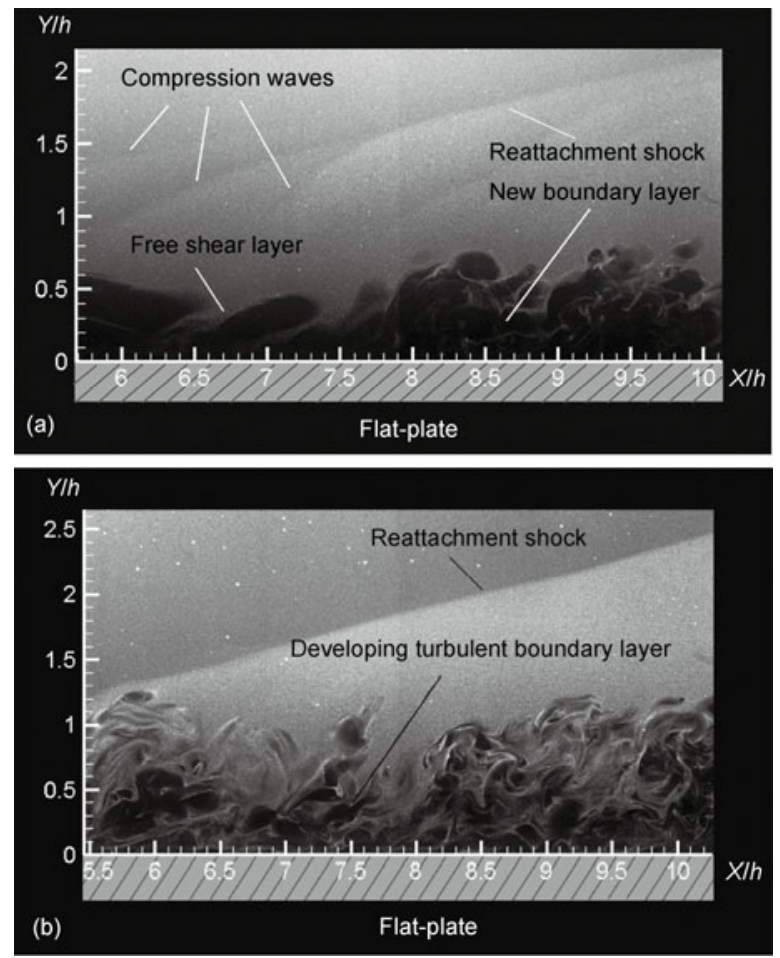

Figure 12 Flow structures around the reattachment region in SLF-BFS (a) and STF-BFS (b), corresponding to Region C in Figure 4.
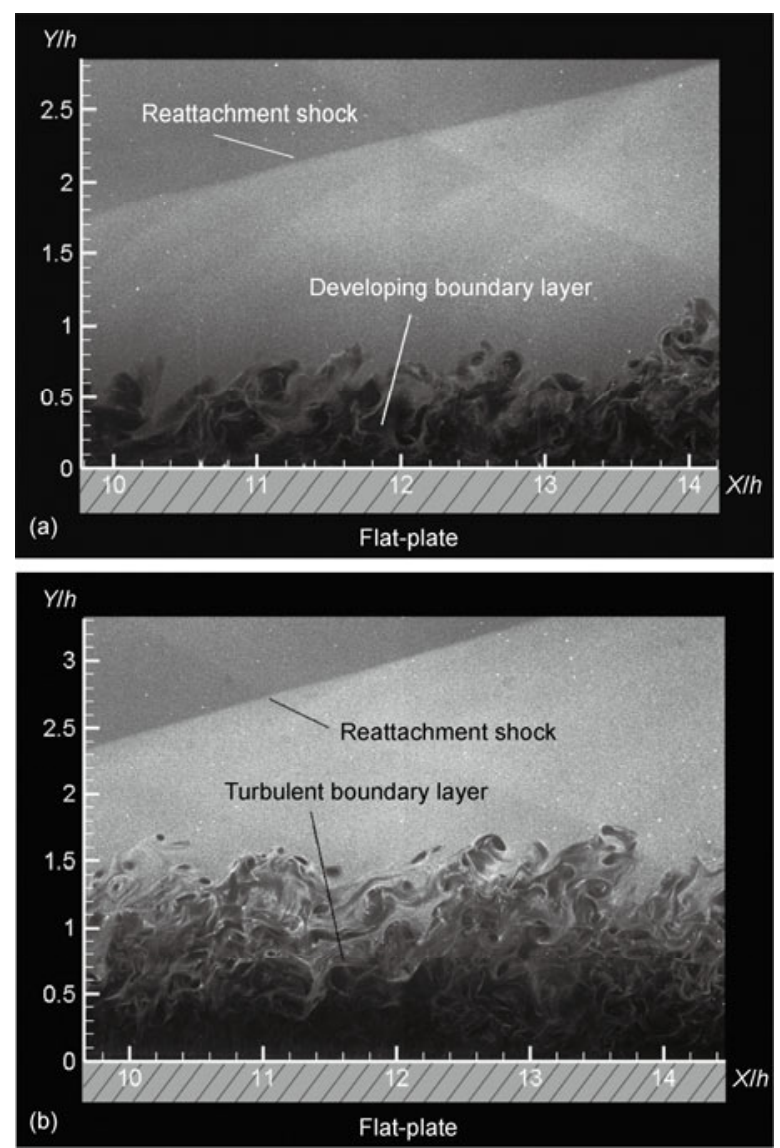

Figure 13 Flow structures of the recovery region in SLF-BFS (a) and STF-BFS (b), corresponding to Region C in Figure 4. 
on the transition and increase of the supersonic laminar boundary layer is quite evident.

Structures of the turbulent boundary layer in Region D evolve rapidly as shown in Figure 13(b). Comparing it with those in Figures 10, 11 and 12, the turbulent boundary layer in Figure 13 contains many more small-scale structures and shedding vortices, which are brought about by the cracking of large-scale structures in the boundary layer upstream.

\section{Conclusions}

Supersonic flow over a BFS is of great importance in many applications as a typical flow field inside engines and on the surface of high speed aircraft. NPLS was adopted in this paper to measure and analyze SLF-BFS and STF-BFS. Compared with traditional techniques such as the Schlieren and shadow, NPLS can distinctly reveal fine flow structures and temporal and spatial evolution with spatial resolution of up to a micron magnitude and temporal resolution of $6 \mathrm{~ns}$, which makes it propitious for studying flow mechanisms.

SLF-BFS is unsteady and prone to separation with a long recirculation region and a new boundary layer after reattachment, rapidly changing into turbulence. In the process of separation, reattachment, transition and redevelopment, the boundary layer clearly evolves and increases at an averaged angle of $4.6^{\circ}$ after reattachment. Compression waves, which are induced by the $K-H$ vortices in the shearing layer, converge into a reattachment shock downstream. The reattachment point locates at about $X=7 h-7.5 h$ according to analysis based on grayscale images. The time-evolution of $K-H$ vortices reveals the impacts from shearing, expansion, reattachment and three-dimensional effects.

Characterized by intensive fluctuations and transport, STF-BFS is more stable and difficult to separate than the SLF-BFS. Its recirculation region is shorter with its reattachment point located at around $3 h-4 h$. Although there are no $K-H$ vortices and obvious compression waves, the same averaging angle of the reattachment shock with the SLF-
BFS indicates the same compression effect from the wall on the separated flow. The boundary layer after reattachment increases slowly while its structures evolve distinctly with small-scale structural shedding. The main characteristics of the large-scale structures before and after reattachment are the inclining and transformation due to the impacts from expansion, near wall viscosity and converse pressure.

1 Shen G X, Ma G Y. The investigation on the properties and structures of starting vortex flow past a back-ward facing step by WBIV technique. Exp Fluids, 1996, 21: 57-65

2 Cohen J M, Bennett J C. An experimental study of the transient flow over a backward-facing step. 1996, AIAA-96-0322

3 Noriyuki F, Tadashi H, Masaya K. An experimental investigation of a large-scale structure of a two-dimensional backward-facing step by using advanced multi-point LDV. Exp Fluids, 2004, 36: 274-281

4 Tinney C E, Ukeiley E L S. A study of a 3-D double backward-facing step. Exp Fluids, 2009, 47: 427-439

5 Neumann J, Wengle H. DNS and LES of passively controlled turbulent backward-facing step flow. Flow Turb Combust, 2003, 71: 297-310

6 Zhao Y X, Yi S H, Tian L F, et al. Supersonic flow imaging via nanopaticles. Sci China Ser E: Tech Sci, 2009, 52: 3640-3648.

7 Ji A C, Liu W M, Song J L, et al. Dynamical creation of fractionalized vortices and vortex lattices. Phys Rev Lett, 2008, 101: 010402

8 Liang Z X, Zhang Z D, Liu W M. Dynamics of a bright soliton in Bose-Einstein condensates with time-dependent atomic scattering length in an expulsive parabolic potential. Phys Rev Lett, 2005, 94: 050402

9 Liu W M, Wu B, Niu Q. Nonlinear effects in interference of Boseinstein condensates. Phys Rev Lett, 2000, 84: 2294-2297

10 Zhao Y X, Yi S H, Tian L F, et al. Density field measurement and approximate reconstruction of supersonic mixing layer. Chin Sci Bull, 2010, 55: 2004-2009

11 Yi S H, He L, Zhao Y X, et al. A flow control study of a supersonic mixing layer via NPLS. Sci China Ser G: Phys Mech Astron, 2009, 52: 2001-2006

12 Zhao Y X. Experimental investigation of spatiotemporal structures of supersonic mixing layer. Doctoral Dissertation. Changsha: National University of Defense Technology, 2008

13 Zhao Y X, Yi S H, He L, et al. The experimental study of interaction between shock wave and turbulence. Chin Sci Bull, 2007, 52: 1297-1301

14 He L, Yi S H, Zhao Y X, et al. Visualization of coherent structures in a supersonic flat-plate boundary layer. Chin Sci Bull, 2011, 56: 489-494

Open Access This article is distributed under the terms of the Creative Commons Attribution License which permits any use, distribution, and reproduction in any medium, provided the original author(s) and source are credited. 